\title{
REDUCED SU(3) CFP's *
}

\author{
D. BRAUNSCHWEIG
}

Dept. of Physics, University of Michigan, Ann Arbor, Michigan 48109, USA

Received 7 July 1977

\section{PROGRAM SUMMARY}

Title of program: REDUCED SU(3) CFPS

\section{Catalogue number: ABKG}

Program obtainable from: CPC Program Library, Queen's University of Belfast, N. Ireland. (See application form in this issue.)

Computer:

AMDAHL $470 \mathrm{~V} / 6$

IBM 360;

\section{Installation:}

Univ. Michigan, Ann Arbor, Michigan

Operating System: MTS

Program language used: FORTRAN

High speed storage required: $197 \mathrm{k}$ words

No. of bits in a word: 32

Overlay structure: none

No. of magnetic tapes required: none

Other peripherals used: card reader, line printer

No. of cards in combined program and test deck: 3655

Card punching code: EBCDIC

CPC Library data deck used:

Catalogue number: AAC*; Title: DATA FOR ABKG

No. of cards in data deck: 7423

Keywords: Nuclear physics, theoretical methods, CFP, SU(3), SU(4), shell model, spectroscopic amplitude, $\alpha$-transfer, pseudo-SU(3).

* Work supported by the U.S. National Science Foundation.

** Block 3 of the program was developed at The Weizmann Institute, Rehovot, Israel.
Nature of physical problem

Reduced SU(3) $x$-particle coefficients of fractional parentage (CFP) are calculated, for any nuclear shell and arbitrary shell model states in an SU(3) $\times$ SU(4) or an SU(3) $\times$ SU(2) scheme. These CFP together with the SU(3) coupling coefficients available with the code of ref. [1] make it possible to perform standard shell model calculations in the SU(3) scheme.

\section{Method of solution}

Raising and lowering operators of SU(3) and SU(4) are used to construct explicitly shell model states of good SU(3) $\times$ SU(4) symmetry [2]. These states are written in terms of Fermion creation operators. Overlaps can then be calculated directly and lengthy recursion is thus avoided.

\section{Restrictions on the complexity of the problem}

Since the size of arrays depends strongly on the nuclear shell and the number of particles, provision is made for easy adjustment of dimensions. However, the number of components of a highest weight state in the many-particle basis should not exceed 200 or else truncation error may accumulate.

\section{Typical running time}

It is a critical function of the nuclear shell, the number of particles, as well as the options selected.

\section{Unusual features of the program}

All the large integer arrays which store numbers that are always less than 256 start with the letter " $L$ ". Therefore in IBM $360 / 370$ or similar operating systems advantage may be taken of the statement IMPLICIT INTEGER*2(L) to save up to $35 \%$ of high speed storage.

\section{References}

[1] Y. Akiyama and J.P. Draayer, Comp. Phys. Comm. 5 (1973) 405.

[2] K.T. Hecht and D. Braunschweig, Nucl. Phys. A244 (1975) 365. 


\section{LONG WRITE-UP}

\section{Introduction}

We describe below a versatile FORTRAN program which calculates reduced coefficients of fractional parentage (CFP's) in the SU(3) X SU(4) scheme. A precise definition of these CFP's (also called triple barred matrix element) can be found in eq. (7) of ref. [1]. These reduced CFP's can be converted to ordinary CFP's in the angular momentum scheme by simple multiplication with an $\mathrm{SU}(3) \supset \mathrm{R}(3)$ reduced Wigner coefficient. The reduced CFP's together with the SU(3) coupling and recoupling coefficients available through the code of Akiyama and Draayer [2] make it possible to perform challenging shell model calculations in the SU(3) scheme [3]. Spectroscopic amplitudes for many-particle transfer reactions are easily obtained from these reduced CFP's [4].

Because of the non-recursive nature of our formulations (see appendix A of ref. [1]), the program calculates with equal ease one or many-particle CFP both at the beginning or in the middle of a shell.

The program was written for applications in the nuclear shell model and it is intended to accommodate the needs of a great variety of users. Care has been exercised to ensure generality.

The program allows calculations to be performed in:

(1) Any major oscillator shell.

(2) Both neutron-proton formalism (NPF) or full spin-isospin formalism (FSIF) are acceptable.

(3) Optimization available for the prolate or oblate limit is obtained by calculating the CFP from the highest weight state (HWS) or lowest weight state (LWS) respectively.

(4) No SU(4) coupling coefficients are needed since both the parent and daughter states can be lowered in SU(4) according to the needs of a particular user.

\section{Method of calculation}

Our states are classified according to the irreducible representation (IR) of $\mathrm{SU}(3) \times \mathrm{SU}(4)$. The subgroup chains used are: $\mathrm{SU}(3) \supset \mathrm{SU}(2) \times \mathrm{U}(1)$ and $\mathrm{SU}(4) \supset$ $\mathrm{SU}(2) \times \mathrm{SU}(2)$. For the meaning of the quantum numbers associated with these chains see refs. [5] and [6].
The availability of the SU(3) $\supset R(3)$ Wigner coefficients makes it possible to perform all calculations in the simpler SU(3) $\supset \mathrm{SU}(2) \times \mathrm{U}(1)$ scheme; since conversion to the physically relevant angular momentum scheme involves only multiplication of a reduced CFP by an SU(3) つ R(3) Wigner coefficient.

Throughout this section we shall assume that the full spin-isospin formalism (FSIF) is used.

Restriction to the simpler neutron-proton formalism (NPF) is obtained by substituting SU(4) by SU(2) and dropping $P^{\prime}, P^{\prime \prime}, T, M_{T}, M_{E}$ while replacing $P$ by $S$.

\subsection{Single particle levels}

For any given major oscillator shell we introduce a single particle index $j$ which is used to number the single particle orbits. See eq. (A.2) of ref. [1]. This is illustrated in fig. 1 for the sd-shell. A one particle state is then written as:

$|j\rangle \equiv a_{\epsilon \Lambda M_{\Lambda} M_{S} M_{T^{M}} M_{E}}|0\rangle$,

where $|0\rangle$ is the vacuum (i.e., closed shell) and $a^{+}$is a Fermion creation operator. Note that we have dropped for the one particle states the SU(3) label since it is implied by the nuclear shell in consideration. Similarly we have dropped the SU(4) label since it is implied by the permutation symmetry [1].

Acting on these single particle states with an $\mathrm{SU}(3)$ or $\mathrm{SU}(4)$ raising (lowering) operator we obtain another state times a normalization coefficient, for example:

$$
\begin{aligned}
& A_{z x}|6\rangle=\sqrt{ } 2|2\rangle, \\
& A_{z x}|18\rangle=0,
\end{aligned}
$$

where $A_{z x}$ is the $\mathrm{SU}(3)$ raising operator that lifts a

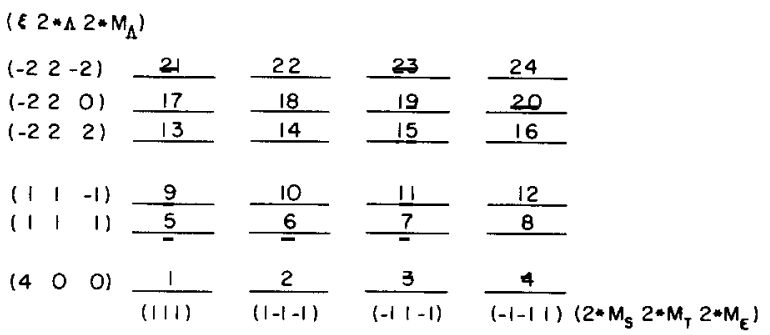

Fig. 1. Numbering of the single particle levels for the sd-shell. The single particle index $j$ runs from 1 to 24 . 
quantum from the $x$-direction into the $z$-direction.

This numbering and the explicit construction of these operators is done in SUBROUTINE LEVELS.

\subsection{Construction of the many-particle basis}

In this section we introduce a many-particle basis in terms of which states of good SU(3) $\times$ SU(4) symmetry can be expanded.

We use the fact that the quantum numbers $\epsilon, M_{\Lambda}$, $M_{S}, M_{T}$ and $M_{E}$ are additive to define as our $n$-particle basis all those states which have a definite value of total $\epsilon, M_{\Lambda}, M_{S}, M_{T}$ and $M_{E}$, that is, all those states of the form

$$
\begin{aligned}
\left|n \in M_{\Lambda} M_{S} M_{T} M_{E}\right\rangle=\left|j_{1}\right\rangle\left|j_{2}\right\rangle \ldots\left|j_{n}\right\rangle \equiv \\
\equiv a_{\epsilon_{1}}^{+} M_{\Lambda_{1}} M_{S_{1}} M_{T_{1}} M_{E_{1}} a_{\epsilon_{2}}^{+} M_{\Lambda_{2}} M_{S_{2}} M_{T_{2}} M_{E_{2}} \\
\quad \ldots a_{\epsilon_{n} M_{\Lambda_{n}} M_{S_{n}} M_{T_{n}} M_{E_{n}}|0\rangle}
\end{aligned}
$$

such that

$\epsilon=\sum_{i=1}^{n} \epsilon_{i} ; \quad M_{\Lambda}=\sum_{i=1}^{n} M_{\Lambda_{i}} ; \quad M_{S}=\sum_{i=1}^{n} M_{S_{i}} ;$

$M_{T}=\sum_{i=1}^{n} M_{T_{i}} ; \quad M_{E}=\sum_{i=1}^{n} M_{E_{i}}$.

Since we write our many-particle states explicitly in terms of Fermion creation operators they become automatically antisymmetric.

This many-particle basis is constructed in SUBROUTINE BASIS.

\subsection{Construction of highest weight states}

A state of highest weight in $\mathrm{SU}(3) \times \mathrm{SU}(4)$ is written as:

$\left|n(\lambda \mu) \alpha\left(P P^{\prime} P^{\prime \prime}\right)\right\rangle_{\mathrm{HW}}$,

where $n=$ number of particles, $(\lambda \mu)=\mathrm{SU}(3)$ irreducible representation, $\alpha=$ inner multiplicity label for $\mathrm{SU}(3)$, needed to distinguish multiple occurrences of $(\lambda \mu)$ in a given space symmetry; $\alpha=1,2, \ldots, \alpha_{\max .},\left(P P^{\prime} P^{\prime \prime}\right)=$ supermultiplet labels.

The labels $\left(P P^{\prime} P^{\prime \prime}\right)$ together with $n$ determine uniquely the spin-isospin symmetry $[\widetilde{f}]$, and since the states are totally antisymmetric they determine as well the space symmetry $[f]$ conjugate to $[\widetilde{f}]$.
This HWS can be expressed as a linear combination of our many-particle basis states which have a definite value of total $\epsilon, M_{\Lambda}, M_{\mathrm{S}}, M_{\mathrm{T}}$ and $M_{\mathrm{E}}$, i.e.:

$\left|n(\lambda \mu) \alpha\left(P P^{\prime} P^{\prime \prime}\right)\right\rangle_{\mathrm{HW}}=\sum_{j=1}^{m} C_{j}\left|n \in M_{\Lambda} M_{S} M_{T} M_{E}\right\rangle_{j}$,

with $\epsilon=2 \lambda+\mu, M_{\Lambda}=\mu / 2, M_{S}=P, M_{T}=P^{\prime}, M_{E}=P^{\prime \prime}$.

The expansion coefficients $C_{j}$ are determined by solving the system of linear equations:

$$
\begin{aligned}
& \left(A_{z x}+A_{z y}+\Lambda_{+}+E_{10}+E_{01}+E_{11}+E_{1-1}+S_{+}\right. \\
& \left.+T_{+}\right)\left|n(\lambda \mu) \alpha\left(P P^{\prime} P^{\prime \prime}\right)\right\rangle_{\mathrm{HW}}=0,
\end{aligned}
$$

where $A_{z x}, A_{z y}$ and $\Lambda_{+}$are the SU(3) raising operators and $E_{10}, E_{01}, E_{11}, E_{1-1}, S_{+}$and $T_{+}$are the SU(4) raising operators (in the notation of ref. [6]).

The number of linearly independent solutions of this homogeneous system of equations is equal to $\alpha_{\max }$. These solutions are orthogonal but otherwise arbitrary. The overall phase of these HWS (in SU(3) and SU(4) is fixed by using the prescriptions given in appendix A of ref. [1]. It should be noted, however, that only non-zero components are considered. The user may compare our phase convention with any other by setting IPRST $=1$ in card 1 of the input data, thus obtaining the explicit form of our HWS.

The system of linear equations is set up in SUBROUTINE RISE.

In the SUBROUTINE TRIANG linearly dependent equations are deleted, and the coefficient matrix is triangularized. In SUBROUTINE HOMEQ a set of linearly independent states is constructed. In SUBROUTINE ORTHO this set of $\alpha_{\max }$ states is orthonormalized.

\subsection{States of lower weight in $S U(4)$}

Since no SU(4) $\supset \mathrm{SU}(2) \times \mathrm{SU}(2)$ Wigner coefficients are available we cannot restrict ourselves to HWS in SU(4). Therefore, we need to construct states which have any of the allowed values of $S$ and $T$ in a given supermultiplet.

This is accomplished by operating on the HWS with the step down operators $O_{\gamma \delta}$ as defined in ref. [6].

$$
\begin{aligned}
& \left|n(\lambda \mu) \alpha\left(P P^{\prime} P^{\prime}\right) \beta S T\right\rangle \\
& \quad=O_{\gamma_{1} \delta_{1}} O_{\gamma_{2} \delta_{2}} \ldots O_{\gamma_{k} \delta_{k}}\left|n(\lambda \mu) \alpha\left(P P^{\prime} P^{\prime \prime}\right)\right\rangle_{\mathrm{HW}},
\end{aligned}
$$


with

$$
\begin{array}{r}
S=P+\sum_{i=1}^{k} \gamma_{i} \text { and } T=P^{\prime}+\sum_{i=1}^{k} \delta_{i} \\
\quad \beta=1,2, \ldots, \beta_{\max } .
\end{array}
$$

$\beta_{\max }$ is the number of linearly independent states with $S$ and $T$ that are contained in the supermultiplet $\left(P P^{\prime} P^{\prime \prime}\right)$. (See eqs. 28-31 of ref. [7].) The choice of operators $O_{\gamma_{i} \delta_{i}}$ in (8) is not unique, however, if $\beta_{\max }=1$, different choices (paths) will lead to the same state except for an overall normalization constant. If $\beta_{\max }>1$ it is necessary to select at least $\beta_{\max }$ different "paths" in order to obtain $\beta_{\max }$ linearly independent solutions. This is illustrated in fig. 2 .

The phase of the state $\left|n(\lambda \mu) \alpha\left(P P^{\prime} P^{\prime \prime}\right) \beta S T\right\rangle$ is determined by the phase of the HWS and the phase of the operators $O_{\gamma \delta}$. Again, as before, this phase can be ascertained by setting IPRST $=1$ card 1 .

$\beta_{\max }$ is calculated in FUNCTION IBETA. The different possible "paths" are found in SUBROUTINE PATHS. The application of the $O_{\gamma \delta}$ operators is performed in SUBROUTINE OXYLOW.

\subsection{The $x$-particle operator}

Since it is simplest to use HWS for both parent and daughter states, the $x$-particle operator which connects them can have arbitrary weight and $x$-particle operators of arbitrary weight must be constructed.

If we write these operators in terms of Fermion creation operators we may take advantage of the fact

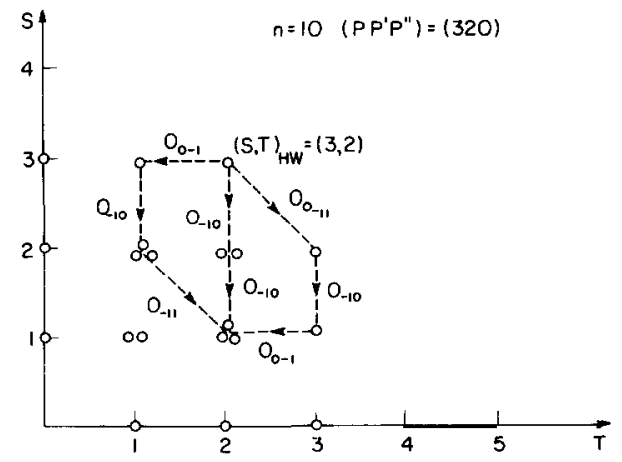

Fig. 2. Three different "paths" that can be taken from the HWS to reach the state with $(S, T)=(1,2)$ by using only lowering operators. that our states were written in a similar fashion and then the same procedure may be used. Explicitly these operators are:

$$
\begin{gathered}
{\left[a_{1}^{+} a_{2}^{+} \ldots a_{x}^{+}\right]^{(\lambda \mu) \alpha \epsilon \Lambda M_{\Lambda}\left(P P^{\prime} P^{\prime \prime}\right) \beta S M_{S} T M_{T}}} \\
=N(\lambda \mu, p, q, r) S_{-}^{\mathrm{s}} T_{-}^{\mathrm{t}} A_{y x}^{\mathrm{r}} O_{y z}^{\mathrm{q}} A_{x z}^{\mathrm{p}} \\
\quad \times\left[a_{1}^{+} a_{2}^{+} \ldots a_{x}^{+}\right]^{(\lambda \mu) \alpha\left(P P^{\prime} P^{\prime \prime}\right) \beta S T} .
\end{gathered}
$$

See ref. [8] for details. Eq. (9) is consistent with the $\mathrm{SU}(3) \supset \mathrm{SU}(2) \times \mathrm{U}(1)$ phase convention of Akiyama and Draayer [2].

For a given oscillator shell and permutation symmetry we have calculated once and for all the lower weight operators as given in eq. (9).

These different sets of operators are supplied with the program and should be used as input data.

In the FSIF the set of operators is specified by $\left[x, 2 P, 2 P^{\prime}, 2 P^{\prime \prime}, N\right]$ while in the NPF they are specified by $[x, 2 S, N]$, where $\left(P P^{\prime} P^{\prime \prime}\right)=$ supermultiplet symmetry of the set, $N=$ oscillator shell, $S=$ spin.

The set of operators we supply and their SU(3) content is summarized in table 1 [9].

\subsection{Calculation of the reduced CFP}

We first evaluate the overlap:

$$
\begin{aligned}
& O_{\Lambda}=\left\langle n+x\left(\lambda_{\mathrm{r}} \mu_{\mathrm{r}}\right) \alpha_{\mathrm{r}}\left(P_{\mathrm{r}} P_{\mathrm{r}}^{\prime} P_{\mathrm{r}}^{\prime \prime}\right) \beta_{\mathrm{r}} S_{\mathrm{r}} T_{\mathrm{r}}\right| \\
& \quad \times\left[a_{1}^{+} a_{2}^{+} \ldots a_{x}^{+}\right]^{(\lambda \mu) \alpha \epsilon \Lambda M_{\Lambda}\left(P P^{\prime} P^{\prime \prime}\right) \beta S M_{S} T M_{T}} \\
& \left.\quad \times \ln \left(\lambda_{\mathrm{c}} \mu_{\mathrm{c}}\right) \alpha_{\mathrm{c}}\left(P_{\mathrm{c}} P_{\mathrm{c}}^{\prime} P_{\mathrm{c}}^{\prime \prime}\right) \beta_{\mathrm{c}} S_{\mathrm{c}} T_{\mathrm{c}}\right\rangle
\end{aligned}
$$

with $\epsilon=2 \lambda_{\mathrm{r}}+\mu_{\mathrm{r}}-2 \lambda_{\mathrm{c}}-\mu_{\mathrm{c}}, M_{\Lambda}=\left(\mu_{\mathrm{r}}-\mu_{\mathrm{c}}\right) / 2$, $M_{S}=P_{\mathrm{r}}-P_{\mathrm{c}}, M_{T}=P_{\mathrm{r}}^{\prime}-P_{\mathrm{c}}^{\prime}$.

If the row and column states are highest weight in SU(3), the total number of admissible values of $\Lambda$, is equal to $\rho_{\max }$, the number of times $\left(\lambda_{\mathrm{r}} \mu_{\mathrm{r}}\right)$ is contained in the Kronecker product $\left(\lambda_{c} \mu_{c}\right) \times(\lambda \mu)$.

The overlap in (10) is easily evaluated since both the HWS and the $x$-particle operator are expressed in terms of the same set of Fermion creation operators. The reduced CFP is then obtained by solving the sys- 
Table 1

The $x$-particle operator set in the FSIF and NPF

\begin{tabular}{lllll}
\hline$x$-particle & SU(3) & SU(2) $\times \mathrm{SU}(2)$ & First card * & Last card * \\
operator & content & content & \\
{$\left[\times 2 \mathrm{P}^{\prime} \mathrm{P}^{\prime} 2 \mathrm{P}^{\prime \prime} \mathrm{N}\right]$} & $(\lambda \mu)$ & $(2 \mathrm{~S} 2 \mathrm{~T})$ &
\end{tabular}

FSIF

\begin{tabular}{lllrr}
\hline$[11111]$ & $(10)$ & $(11)$ & 1 & 13 \\
{$[22001]$} & $(20)$ & $(20)(02)$ & 14 & 44 \\
{$[22221]$} & $(01)$ & $(22)(00)$ & 45 & 74 \\
{$[311-11]$} & $(30)$ & $(11)$ & 75 & 121 \\
{$[40001]$} & $(40)$ & $(00)$ & 122 & 150 \\
{$[11112]$} & $(20)$ & $(11)$ & 151 & 172 \\
{$[22002]$} & $(40)(02)$ & $(20)(02)$ & 173 & 310 \\
{$[22222]$} & $(21)$ & $(22)(00)$ & 311 & 451 \\
{$[311-12]$} & $(60)(22)(00)$ & $(11)$ & 452 & 967 \\
{$[40002]$} & $(80)(42)$ & $(00)$ & 968 & 1875 \\
{$[11113]$} & $(30)$ & $(11)$ & 1876 & 1908 \\
{$[22003]$} & $(60)(22)$ & $(20)(02)$ & 1909 & 2363 \\
{$[22223]$} & $(41)(03)$ & $(22)(00)$ & 2364 & 2881 \\
{$[311-13]$} & $(90)$ & $(11)$ & 2882 & 3769 \\
{$[40003]$} & $(120)$ & $(00)$ & 3770 & 6044
\end{tabular}

$N P F$

\begin{tabular}{llll}
{$[\mathrm{x} 2 \mathrm{SN}]$} & $(\lambda \mu)$ & & \\
\hline$[111]$ & $(10)$ & 6045 & 6056 \\
{$[201]$} & $(20)$ & 6057 & 6068 \\
{$[221]$} & $(01)$ & 6069 & 6081 \\
{$[112]$} & $(20)$ & 6082 & 6094 \\
{$[202]$} & $(40)(02)$ & 6095 & 6123 \\
{$[222]$} & $(21)$ & 6124 & 6164 \\
{$[113]$} & $(30)$ & 6165 & 6186 \\
{$[203]$} & $(60)(22)$ & 6187 & 6259 \\
{$[223]$} & $(41)(03)$ & 6260 & 6389
\end{tabular}

* The card numbers refer to the sequence numbers in deck AAC*

tem of linear equations:

$$
\begin{aligned}
& O_{\Lambda}=\sum_{\rho=1}^{\rho_{\max }}\left\langle\left(\lambda_{\mathrm{c}} \mu_{\mathrm{c}}\right) \epsilon_{\mathrm{c}} \Lambda_{\mathrm{c}} ;(\lambda \mu) \epsilon \Lambda \|\left(\lambda_{\mathrm{r}} \mu_{\mathrm{r}}\right) \epsilon_{\mathrm{r}} \Lambda_{\mathrm{r}}\right\rangle_{\rho} \\
& \left\langle\Lambda_{\mathrm{c}} \Lambda_{\mathrm{c}} \Lambda M_{\Lambda} \mid \Lambda_{\mathrm{r}} \Lambda_{\mathrm{r}}\right\rangle\left\langle S_{\mathrm{c}} S_{\mathrm{c}} S M_{S} \mid S_{\mathrm{r}} S_{\mathrm{r}}\right\rangle \\
& \left\langle T_{\mathrm{c}} T_{\mathrm{c}} T M_{T} \mid T_{\mathrm{r}} T_{\mathrm{r}}\right\rangle\left\langle n+x\left(\lambda_{\mathrm{r}} \mu_{\mathrm{r}}\right) \alpha_{\mathrm{r}}\left(P_{\mathrm{r}} P_{\mathrm{r}}^{\prime} P_{\mathrm{r}}^{\prime \prime}\right) \beta_{\mathrm{r}} S_{\mathrm{r}} T_{\mathrm{r}}\right| \\
& \|\left[a_{1}^{+} a_{2}^{+} \ldots a_{x}^{+}\right]^{(\lambda \mu) \alpha\left(P P^{\prime} P^{\prime \prime}\right) \beta S \rho^{\prime} \|} \\
& \left|n\left(\lambda_{\mathrm{c}} \mu_{\mathrm{c}}\right) \alpha_{\mathrm{c}}\left(P_{\mathrm{c}} P_{\mathrm{c}}^{\prime} P_{\mathrm{c}}^{\prime \prime}\right) \beta_{\mathrm{c}} S_{\mathrm{c}} T_{\mathrm{c}}\right\rangle,
\end{aligned}
$$

where $\epsilon_{\mathrm{c}}=2 \lambda_{\mathrm{c}}+\mu_{\mathrm{c}}, \Lambda_{\mathrm{c}}=\mu_{\mathrm{c}} / 2, \epsilon_{\mathrm{r}}=2 \lambda_{\mathrm{r}}+\mu_{\mathrm{r}}, \Lambda_{\mathrm{r}}=\mu_{\mathrm{r}} / 2$, $\rho=1,2, \ldots, \rho_{\max }$.
The triple barred quantity in eq. (11) is the reduced CFP. This reduced CFP is multiplied by three ordinary SU(2) Wigner coefficients and a reduced SU(3) $\supset$ $\mathrm{SU}(2) \times \mathrm{U}(1)$ Wigner.

\section{Structure of the program}

The program is divided into five blocks as shown in fig. 3. Each block performs a logically different function and contains several subroutines. Communication between these subroutines is established through labeled COMMON blocks.

All the input to the program is read in the MAIN 


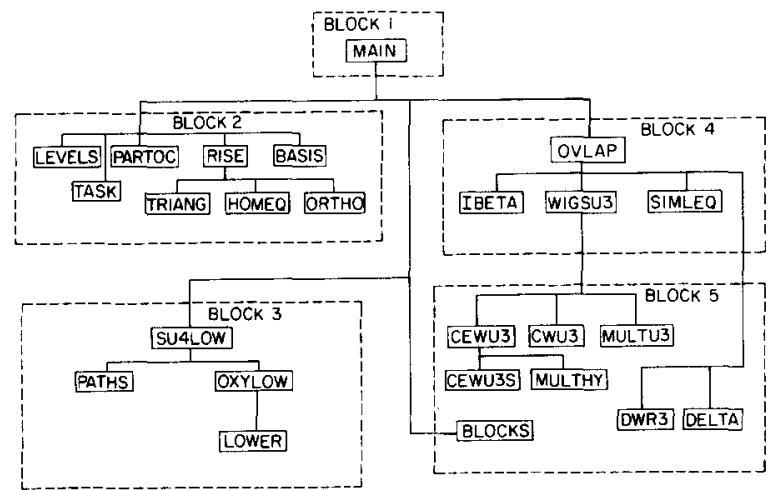

Fig. 3. Flow diagram for program Reduced SU(3) CFPS.

ROUTINE which is the only one contained in BLOCK 1 (see sec. 4).

In BLOCK 2 the HWS are constructed following the method outlined in sec. 2.

In BLOCK 3 the stepping down in SU(4) is done. This block is needed if and only if the FSIF is used and furthermore the states of interest are not highest weight in SU(4). Appreciable saving in storage is gained if this block is deleted for calculations involv. ing only the NPF.

In BLOCK 4 the reduced CFP's are calculated and printed out at the end of SUBROUTINE OVLAP.

BLOCK 5 contains the subroutines needed to calculate the reduced $\mathrm{SU}(3) \supset \mathrm{SU}(2) \times \mathrm{U}(1)$ Wigners as well as the SU(2) Wigners. These subroutines are taken without change from PART 1 of the SU(3) PACKAGE of ref. [2].

\section{Input/output}

The input to the program is of two types. Fig. 4 illustrates the structure of the input data deck.

\subsection{Input of type I}

Input of this type is provided in a separate data deck, catalogue number AAC*, and allows the user to select a particular use of the program. By using different data sets of the type I the user may calculate 1 particle CFP, 2 particle CFP, 3 particle CFP, or 4 particle CFP (alpha transfer) in either the $p$, sd, or fp shells, in the FSIF or NPF.

Input of type I consists of two blocks of input

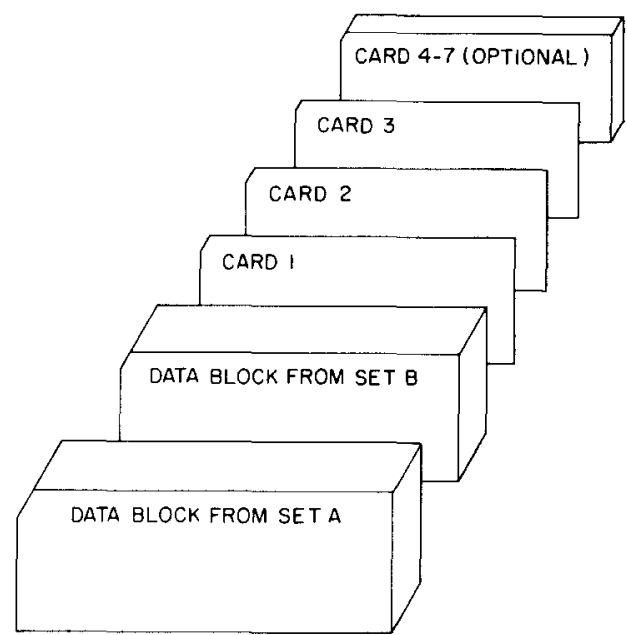

Fig. 4. Structure of the input data deck.

data, the first block to be selected from set $A$ and the second block from set $B$. Combinations which are not allowed are detected by the program and a relevant message printed.

\subsubsection{Data set $A$}

Data blocks of set A contain all the information needed to fully describe the $x$-particle operator.

We explain below the meaning of all quantities read in for set $A$.

MPOPX $=$ value of $x$ in $\left[x, 2 P, 2 P^{\prime}, 2 P^{\prime \prime}, N\right]$ or $[x, 2 S, N]$.

IOPMX $=$ total number of $x$-particle operators with symmetry $\left(P P^{\prime} P^{\prime \prime}\right)$.

MULARN = number of SU(3) IR considered times $\operatorname{dim}_{\mathrm{SU}(4)}[\tilde{f}]$.

NCFMX = total number of components of all the different $x$-particle operators.

NSHELL $\quad=N+1$.

NEUPRO $=1$ for NPF.

$=2$ for FSIF.

MULAMN(I) = sequence number assigned to the first $x$-particle operator which has SU(3) symmetry number I (reading from left to right in the second column of table 1).

NXOPMN(I) = starting address for the $x$-particle operator of sequence number $I$ in array CFOPX.

LEPXOP(I) $=\epsilon$ of $x$-particle operator with sequenc no. I. 
LAMXOP(I) $=\Lambda$ of $x$-particle operator with sequence no. I.

LMMXOP(I) $=M_{\Lambda}$ of $x$-particle operator with sequence no. I.

LSXOP(I) $=S$ of $x$-particle operator with sequence no. I.

LTXOP(I) $=T$ of $x$-particle operator with sequence no. I.

LSMXOP(I) $=M_{S}$ of $x$-particle operator with sequence no. I.

LTMXOP(I) $=M_{T}$ of $x$-particle operator with sequence no. I.

LCXOP(I) = stores the components of the $x$-particle operator.

CFOPX(I) $=$ stores the coefficient of each component of the $x$-particle operator.

\subsubsection{Data set $B$}

Data blocks of set $B$ contain a table for the reduction $\mathrm{U}(n) \supset \mathrm{SU}(3)$ where $n=$ NSHELL $*$ (NSHELL $+1) / 2$. Only those IR of U(n) up to half full shell and which have not more than four columns are tabulated. For the fp shell we include only those SU(3) IR which have $\alpha_{\max } \leqslant 10$. This is summarized in table 2 . The same data block is used for the FSIF and NPF. We explain below the meaning of the quantities which are read in.

ISAYRD $=$ NSHELL $*($ NSHELL +1$) / 2$.

NUNTOT $=$ number of U(n) IR.

NSUTOT = number of SU(3) IR.

LPARMN(I) = starting address in array LUNREP of $\mathrm{U}(n)$ IR with I boxes.

LUNREP = stores the number of boxes in each column of the tableaux of $U(n)$.

Table 2

Data blocks of set B. For the fp shell only those SU(3) IR which have $\alpha_{\max }<10$ is included.

\begin{tabular}{llll}
\hline $\begin{array}{l}\text { Space } \\
\text { symmetry } \\
\mathrm{U}(n)\end{array}$ & $\begin{array}{l}\text { Restrictions } \\
\text { on SU(3) } \\
\text { content }\end{array}$ & $\begin{array}{l}\text { First } \\
\text { card * }\end{array}$ & $\begin{array}{l}\text { Last } \\
\text { card }\end{array}$ \\
\hline $\mathrm{U}(3)$ & None & 6390 & 6399 \\
$\mathrm{U}(6)$ & None & 6400 & 6525 \\
$\mathrm{U}(10)$ & $\alpha_{\max }<10$ & 6526 & 7423 \\
\hline
\end{tabular}

* The card numbers refer to the sequence numbers in deck AAC*.
LSU3MN(I) = starting address for the SU(3) content of U( $n)$ IR number I in LSU3CF and LSU3RP.

LSU3CF(I) = number of times that SU(3) IR number I is contained in the IR of $U(n)$ connected by LSU3MN.

LSU3RP(I, J) $=(\lambda \mu)$ of SU(3) IR number I.

$J=1$ gives $\lambda$.

$J=2$ gives $\mu$.

\subsection{Input of type II}

This data must be provided by the user. In its simplest form it contains only three cards. Seven cards is the maximum.

Card 1: IPRST, IPRLVL, IPRTSK FORMAT (1614)

The variables in Card 1 control only the extent of the printing performed. Its use is spelled out in comment statements in SUBROUTINES OVLAP. Leaving Card 1 blank simply prints the reduced CFP's calculated.

Card 2: IREPMN, IREPMX, LOHI FORMAT (1614)

IREPMN and IREPMX define the first and last SU(3) symmetry (in the order of table 1) of the $x$-particle operator to be used.

LOHI determines whether the overlap is to be calculated from HSW or LWS in SU(3). For prolate deformations $(\lambda \geqslant \mu)$ use LOHI $=0$ while for oblate deformations $(\lambda<\mu)$ use LOHI $=1$.

Card 3: $\{[\operatorname{LINBUF}(\mathrm{I}, J), \mathrm{I}=1, \operatorname{IMAX}], J=1,2\}$ FORMAT (1614)

IMAX $=4$ for NPF.

IMAX $=8$ for FSIF.

$J=1$ for row (bra) states.

$J=2$ for column (ket) states.

This card defines the range of the calculation. The meaning and allowed values for LINBUF are summarized in table 3.

In order to achieve the maximum of versatility some of the elements of the array LINBUF can be given some reserved values which will give to the associated variable not a specific value but rather a set of values.

Reserved value -111: If given to an element of LINBUF the associated variable is allowed to take all possible values consistent with the other quantum numbers, e.g., $\operatorname{LINBUF}(2,1)=-111$ specifies that all the allowed values of $\epsilon=2 \lambda+\mu$ will be considered. 
Table 3

$\{[\operatorname{LINBUF}(\mathrm{I}, J), \mathrm{I}=1,8], J=1,2\}$

\begin{tabular}{|c|c|c|c|c|c|c|c|c|}
\hline I & 1 & 2 & 3 & 4 & 5 & 6 & 7 & 8 \\
\hline $\begin{array}{l}\text { Associated } \\
\text { variable }\end{array}$ & $\begin{array}{l}\text { number } \\
\text { of part. }\end{array}$ & $2 \lambda+\mu$ & $\lambda+2 \mu$ & $2 \mathrm{P}^{*}$ & $2 \mathrm{P}^{\prime}$ & $2 P^{\prime \prime}$ & $2 S$ & $2 \mathrm{~T}$ \\
\hline Integer & Yes & Yes & Yes & Yes & Yes & Yes & Yes & Yes \\
\hline $\begin{array}{l}\text { Reserved } \\
\text { value }-111\end{array}$ & No & Yes & Yes & Yes & Yes & Yes & Yes & Yes \\
\hline $\begin{array}{l}\text { Reserved } \\
\text { value }-222\end{array}$ & No & Yes & Yes & No & No & No & No & No \\
\hline $\begin{array}{l}\text { Reserved } \\
\text { value }-333\end{array}$ & No & No & No & No & No & No & Yes & Yes \\
\hline
\end{tabular}

This is only needed for FSIF

* In the NPF $2 P$ should be understood to be $2 S$.

Reserved value -222: If given to an element of LINBUF the associated variable must be defined in cards $4-7$. This is useful if one wishes to consider only a set of very specific values for the associated variable. Reserved value -333: Can be given only to LINBUF $(7, J)$ and $\operatorname{LINBUF}(8, J)$. It gives to the associated variable the highest weight value consistent with $\left(P P^{\prime} P^{\prime \prime}\right)$. This is useful if the reserved value -111 was given to another element of LINBUF.

Card 4-7: FORMAT (1614) Are needed only if in card 3 the reserved value -222 was used. The number of cards needed is equal to the number of times -222 appeared in card 3.

Cards 4-7 refer to the associated variable connected respectively with the first, second, third and fourth appearances of -222 in card 3 (reading from left to right).

The first integer on these indicates the number of different values to be assigned to the associated variable. The rest of the card must then contain the values of the associated variable.

\subsection{Output}

The output is controlled by the value of the variables in Card 1. It should be noted that for all the quantum numbers which can take half-integer values, twice its magnitude is printed.
Table 4

Parameters of the size of arrays in the shell model

\begin{tabular}{llrrr}
\hline $\begin{array}{l}\text { Common } \\
\text { block }\end{array}$ & Param- & \multicolumn{3}{c}{ Oscillator shell } \\
\cline { 3 - 5 } & eter & \multicolumn{2}{c}{ p } & \multicolumn{1}{c}{ sd } \\
\hline CLVL & NS1 & 12 & 24 & 40 \\
CPRT & NS2 & 5 & 17 & 33 \\
& NP1 & 50 & 100 & 200 \\
CUSU & NS3 & 36 & 452 & 2108 \\
& NS4 & 8 & 16 & 24 \\
& NS5 & 20 & 114 & 528 \\
& NS6 & 20 & 1220 & 9564 \\
CSHM & NP2 & 100 & 150 & 150 \\
& NP3 & 80 & 100 & 100 \\
CINP & NP4 & 20 & 20 & 20 \\
& NP5 & 100 & 100 & 100 \\
CXOP & NP6 & 112 & 3852 & 4004 \\
& NP7 & 44 & 228 & 452 \\
CBAS & NP9 & 8000 & 15000 & 15000 \\
& NP10 & 100 & 200 & 200 \\
& NP11 & 1000 & 2000 & 3000 \\
CROW & NP12 & 20 & 20 & 20 \\
& NP13 & 500 & 500 & 500 \\
& NP14 & 5000 & 5000 & 5000 \\
COXY & NP15 & 50 & 100 & 200 \\
& NP16 & 1000 & 2000 & 4000 \\
CPAT & NP17 & 100 & 100 & 100 \\
& NP18 & 1000 & 1000 & 1000 \\
CLOW & NP19 & 100 & 100 & 200 \\
& NP20 & 1000 & 1000 & 2000 \\
\hline
\end{tabular}




\section{Program modifications}

A complicating feature of the shell model is that the size of the calculations increases very rapidly from one shell to the next; and within a shell with increasing particle number.

Therefore, the size of the arrays should be fixed by the user in accordance with particular needs.

Arrays whose size need to be modified are dimensioned in COMMON blocks. Their size is determined by the parameters in table 4 and comment statements relevant to the COMMON blocks.

Parameters which begin with "NS" get values which depend only on the oscillator shell under consideration.

Parameters which begin with "NP" should be given a tentative value (suggested in table 4). In the event that the dimension is not large enough, a relevant message will be printed and the user asked to increase its value.

As provided by us the program has dimensions sufficient for the fp-shell.

Considerable saving of high speed storage (up to $35 \%$ ) can be achieved by using half-length words for all the integer arrays which begin with the letter "L". In IBM or similar machines this is done by including as the first statement of each subroutine IMPLICIT INTEGER $* 2(L)$.

\section{Test run}

Because of the versatility of the code we cannot reproduce here sample outputs for all the options. We have selected the case of symmetrically coupled two particle CFP's in the fp shell within the FSIF. The daughter state chosen is

$\left|n=6(\lambda \mu)=(120)\left(P P^{\prime} P^{\prime \prime}\right)=(100) S=1 T=0\right\rangle$.

All the $(2 \lambda+\mu)=18$ parent states are calculated.

Below we give the input deck required for this run:

DATA SET A: [22003]

CARDS $1909-2363$ of data deck AAC*

DATA SET B: U(10) つ SU(3)

CARDS $6526-7423$ of data deck AAC*

CARD 2: 11

\section{Acknowledgments}

The author wishes to express his indebtedness to Prof. K.T. Hecht for proposing this work and for continuous fruitful discussions throughout its development. The author is especially grateful to Prof. Hecht for his teachings in the methods of group theory.

\section{References}

[1] K.T. Hecht and D. Braunschweig, Nucl. Phys. A244 (1975) 365.

[2] J.P. Draayer and Y. Akiyama, J. Math. Phys. 14 (1973) 1904

Y. Akiyama and J.P. Draayer, Computer Phys. Commun. 5 (1973) 405.

[3] D. Braunschweig and K.T. Hecht, Bull. Aps. 22 (1977) EE8; J.P. Draayer, Nucl. Phys. A216 (1973) 457; Y. Akiyama, A. Arima, T. Sebe, Nucl. Phys. A138 (1969) 273.

M. Conze, H. Feldmeier, P. Manakos, Phys. Lett. 43B (1973) 101;

R.D. Ratna Raju, J.P. Draayer, K.T. Hecht, Nucl. Phys. A202 (1973) 433;

[4] J.P. Draayer, Nucl. Phys. A237 (1975) 157;

N. Anantaraman, H.E. Gove, J. Toke, J.P. Draayer, Nucl. Phys. A279 (1977) 474.

[5] M. Harvey, Adv. Nucl. Phys. 1 (1968) 67.

[6] K.T. Hecht and S.C. Pang, J. Math. Phys. 10 (1969) 1571.

[7] G. Racah, Rev. Mod. Phys. 21 (1949) 494.

[8] K.T. Hecht, Nucl. Phys. 62 (1965) 1.

[9] In a future publication we will make available the code which constructs the $x$-particle operators.

CARD 3: $\quad 62412 \quad 2 \quad 0 \quad 0 \quad 2 \quad 0 \quad 4$ 18-111-111-111-111-111-111 FORMAT(1614) 


\section{TEST RUN OUTPUT}

\begin{tabular}{|c|c|c|c|c|c|c|c|c|c|c|c|c|c|c|c|}
\hline & & & & $1 S T$ & 8) & & $\left(\begin{array}{lll}S & T & E\end{array}\right)$ & & 15 & T E) & & $1 \mathrm{ST}$ & E) & & \\
\hline$E P S=-3$ & LADTR $=3$ & LELDTH=-3 & ----37 & 111 & 1) & -----38 & $(1-1-1)$ & ------39 & $1-1$ & $1-1)$ & ------40 & $(-1-1$ & 1) & ----- & $\left(\begin{array}{llll}0 & 0 & 3\end{array}\right)$ \\
\hline EPS $=-3$ & LHDTU $=3$ & LELDT $W=-1$ & ----33 & $(11$ & 1) & $---2--34$ & $(1-1-1)$ & ------35 & $(-1$ & $1-11$ & -----36 & $(-1-1)$ & 1) & ----- & $\left(\begin{array}{lll}0 & 1 & 2\end{array}\right)$ \\
\hline EPS $=-3$ & LADTW $=3$ & $\operatorname{LULDT}=1$ & ----29 & ( 111 & 1) & $-\cdots-30$ & $(1-1-1)$ & $-\cdots+31$ & {$[-1$} & $\mid-1)$ & --+---32 & $1-1-1$ & 1) & ---- & $\left(\begin{array}{lll}0 & 2 & 1\end{array}\right)$ \\
\hline$E P S=-3$ & ISDTH $=3$ & LGLDTH $=3$ & ----25 & 111 & 1) & ----26 & $(1-1-1)$ & ----27 & $1-1$ & $1-1)$ & ------28 & $(-1-1)$ & 1) & ---- & $(03 n)$ \\
\hline$E P S=0$ & LHDTH=2 & I $L L D T R=-2$ & $-\cdots-21$ & $\left(\begin{array}{ll}1 & 1\end{array}\right.$ & 1) & $-\cdots--22$ & $(1-1-1)$ & $\cdots---23$ & $1-1$ & $(1-1)$ & ------24 & $(-1-1$ & 1) & $-\ldots+$ & $(1 c 2)$ \\
\hline$E P S=0$ & LMDTW = 2 & IMLTH = ? & ----17 & ( 111 & 1) & $-\ldots--18$ & $(1-1-1)$ & -----19 & $1-1$ & $(-1)$ & -----20 & $(-1-1)$ & 1) & --- & $(191)$ \\
\hline PPS $=0$ & $\operatorname{LMDTN}=2$ & LALDIN $=2$ & ----13 & 111 & 1) & $\ldots-14$ & $(1-1-1)$ & $\cdots-15$ & $1-1$ & $1-1)$ & $\ldots--9-16$ & $1-1-1$ & 1) & $\ldots$ & $(120)$ \\
\hline EPS $=3$ & IMDTY $=1$ & I HID $28=-9$ & ---9 & 111 & 1) & -----10 & $(1-1-1)$ & ----11 & $1-1$ & $(-1)$ & ----12 & $(-1-1)$ & 1) & ---- & $\left(\begin{array}{lll}2 & 0 & 1\end{array}\right)$ \\
\hline$E P S=3$ & $\operatorname{LADTM}=1$ & IGLDTH $=1$ & $\cdots 5$ & $(1)$ & 1) & $\cdots-6$ & $(1-1-1)$ & 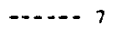 & $(-1$ & $(-1)$ & $\ldots-18$ & $(-1-1)$ & 1) & $\cdots$ & $(210)$ \\
\hline
\end{tabular}

\section{********* SCOPE OP THE CALCULATION FOR DAUGHTER STATES **********}

POR $(P S=2 P T=O P E=0) \quad A N D(5, T)=\left(\begin{array}{lll}2 & 0\end{array}\right)$

$2(120)$

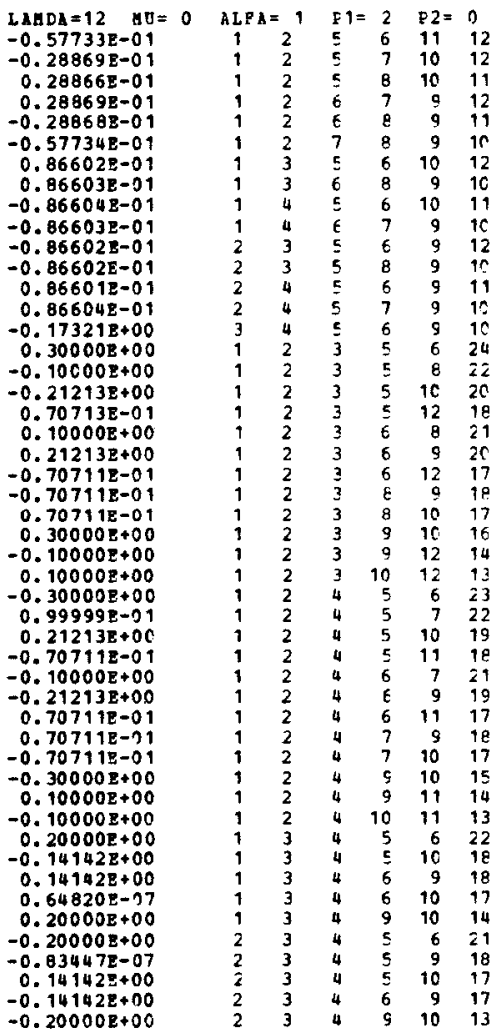



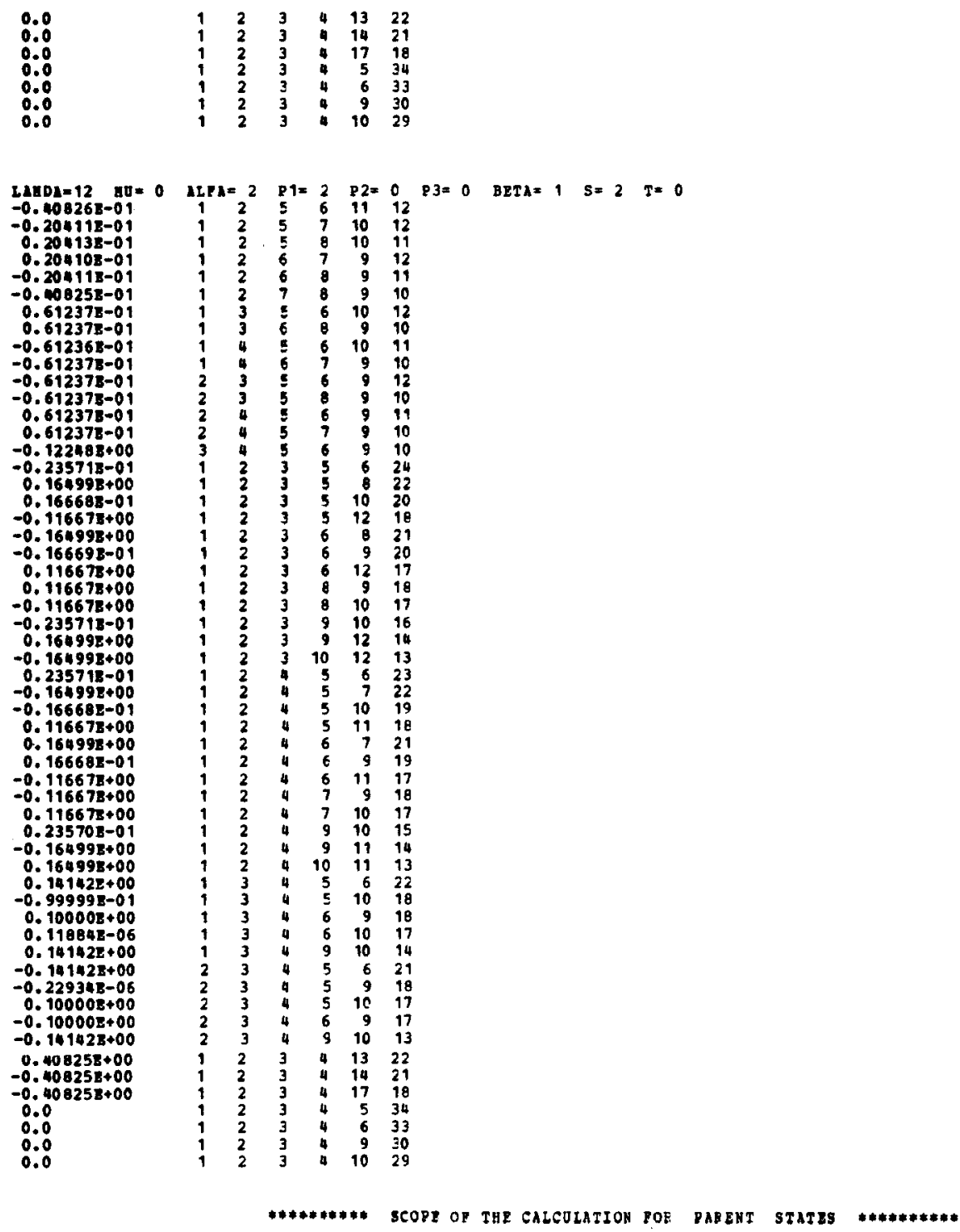

$P O R \quad(P S=O P T=O B P=0, \quad$ AND $\quad(5, T)=(C)$

TOR (PSE 2 PT= $2 P E=C)$ AHD $(S, T)=(22)(20)(P 2)$

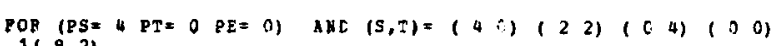

$1(82)$

IAHDA $=8 \quad M O=2 \quad A L F A=1 \quad P 1=0 \quad P 2=C \quad P 3=0 \quad B E T A=1 \quad S=0 \quad T=0$ $-0.174088+00$
$0.17408 E+00$

$-0.174088+00$

$-0.17408 \mathrm{~s}+00$

$3.174088+00$

$-0.175278000$

$-0.452272+00$

$-0.45227 z+00$

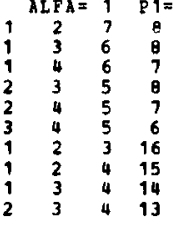




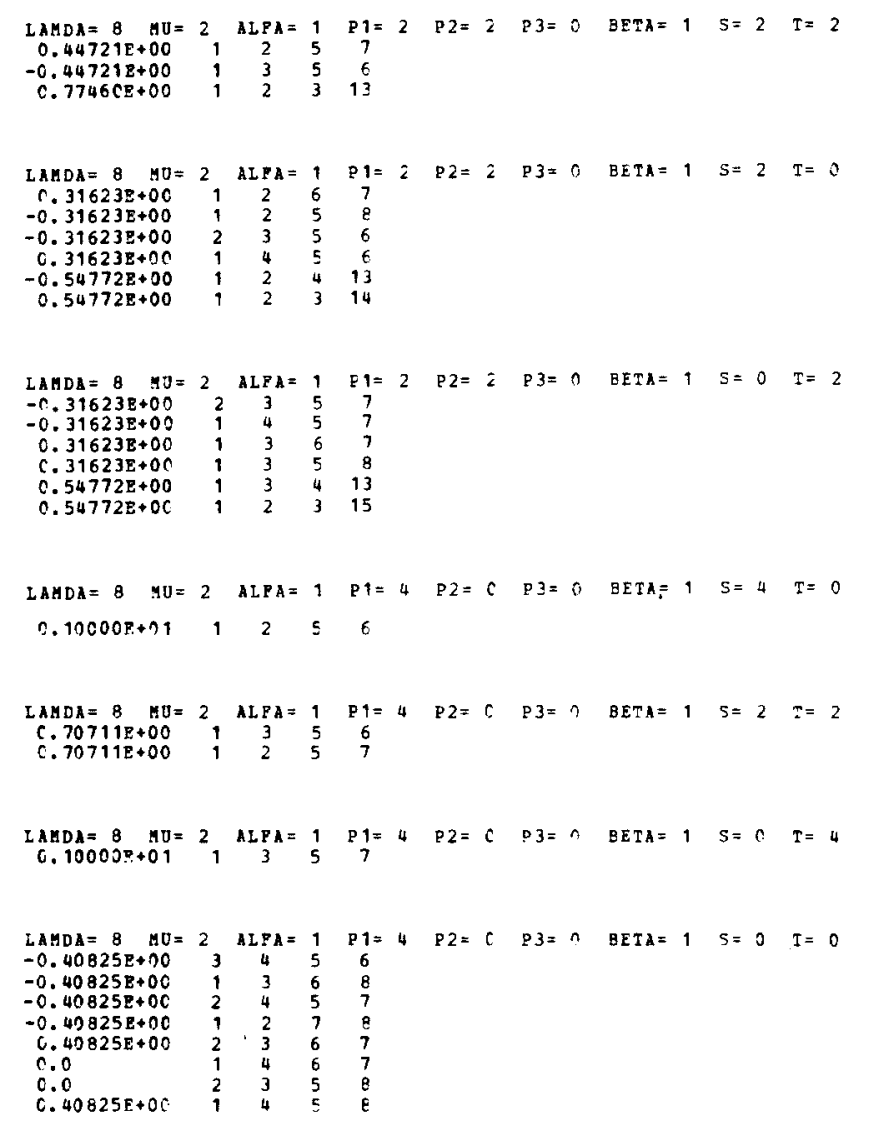

\begin{tabular}{|c|c|c|c|c|c|c|c|c|c|c|c|c|c|c|c|c|c|c|c|c|c|c|c|c|c|c|c|c|}
\hline$<$ & N & (LA & MU) & ALPA & ( PS & $S$ ET & FE ) & BETA & $\leq \mathrm{I}$ & III & (LA & (I) & $S T$ & T PO & III & $N-i$ & (IA & MU) & A L FA & $(\mathrm{P}$ & S $P$ & PT & PE I & BETA & $s$ & $>=$ & CPP & $(C P P * * 2)$ \\
\hline$<$ & 6 & 112 & 0) & 1 & 2 & 0 & ( ) & 1 & 20 & I I I & 16 & 0) & 2 & 1 & III & 4 & 18 & 2) & 9 & & 0 & 0 & & 1 & 00 & $>=$ & 1.09546 & $(1,20003)$ \\
\hline$<$ & 6 & 112 & o) & $i$ & $i$ & 0 & c i & $i$ & 20 & III & 16 & 0) & 20 & 01 & III & 4 & & & 1 & i & 2 & 2 & & & & $>$ & & \\
\hline$<$ & 6 & 112 & oi & $i$ & $i$ & c & 63 & 1 & 26 & III & $i \epsilon$ & of & 20 & 01 & III & 4 & $i \theta$ & 2) & 1 & & 4 & 0 & & 1 & & $>=$ & & \\
\hline$<$ & 6 & 112 & $0 i$ & $i$ & $i$ & $c$ & c) & $i$ & 20 & III & $i 6$ & o) & 20 & 1 & III & 4 & 18 & 2) & $i$ & i & 4 & 0 & 0 & 1 & 00 & $5=$ & $=-0.23355$ & 55) \\
\hline$<$ & 6 & 112 & oi & $i$ & i & 0 & cj & 1 & 20 & III & 16 & o) & 62 & 21 & 111 & 4 & ( 8 & 2) & $\uparrow$ & i & 2 & 2 & & 1 & & $>=$ & $=0.44313$ & 36) \\
\hline$<$ & 6 & (12 & 0) & 1 & i & 0 & ci & 1 & $2 c$ & III & 16 & 0) & ? 2 & 21 & III & 4 & 18 & 2) & 1 & i & 4 & $?$ & & 1 & 2 & $=$ & $=-0$. & \\
\hline$<$ & 6 & (112 & c) & $i$ & $i$ & c & c & $i$ & 20 & III & 12 & 2) & 20 & 61 & III & 4 & 18 & 2) & 1 & & 0 & 0 & & $i$ & 0 & & $=-0$. & \\
\hline$<$ & 6 & 112 & ()) & 1 & $i$ & $c$ & ( ) & 1 & 20 & III & 12 & 2) & 20 & 61 & III & 4 & 18 & 2) & 1 & ( & 2 & 2 & & $i$ & & $>=$ & $=-0$. & \\
\hline$<$ & 6 & i12 & of & 1 & $i$ & c & ( 1 & 1 & 20 & III & 12 & 2) & 20 & 1 & III & 4 & i 8 & & 1 & & 4 & 0 & & 1 & & $=$ & $=0$ & \\
\hline$<$ & 6 & (12 & of & $i$ & i & i & i) & $i$ & $2 c$ & III & 12 & 2) & 26 & 6 & III & 4 & 18 & 2) & 1 & & 4 & 0 & & $i$ & & $=$ & & \\
\hline$<$ & 6 & (12 & 0) & $i$ & $i$ & 0 & (i) & 1 & 20 & III & 12 & 2) & 02 & 21 & III & 4 & 18 & 21 & $i$ & $i$ & 2 & 2 & & 1 & & $=$ & $=0.2$ & 0001 \\
\hline$<$ & 6 & (12 & c) & 1 & $i$ & c & $c j$ & 1 & 28 & III & 12 & 2) & 0 & 21 & III & 4 & 18 & & 1 & & 4 & 3 & & 1 & & $=$ & $=-0$ & \\
\hline$<$ & 6 & (12 & 3 & 2 & $i$ & 0 & $c i$ & 1 & $\approx 0$ & III & 16 & $0)$ & 20 & 1 & III & 4 & 18 & 2) & 1 & & 0 & 0 & & 1 & 00 & $=$ & $=-0$. & \\
\hline$<$ & 6 & (12 & c) & 2 & $i$ & 0 & 01 & 1 & $\approx n$ & III & 16 & 0) & 20 & 01 & III & 4 & 18 & 2 & 1 & i & 2 & 2 & & 1 & & 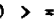 & $=-0$. & \\
\hline$<$ & 6 & i12 & c) & 2 & $i$ & 0 & $0 j$ & 1 & & II I & $i 6$ & $0 j$ & 20 & 6) 1 & III & 4 & i 8 & & 1 & & & & & 1 & & 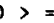 & & \\
\hline$<$ & 6 & i12 & 0) & 2 & $i$ & $c$ & ( i & 1 & 20 & III & 16 & c) & $2 n$ & 1 & III & 4 & 18 & 2) & 1 & i & 4 & 0 & & 1 & 00 & $>=$ & 0.0 & 3031 \\
\hline$<$ & 6 & 112 & () & 2 & 1 & c & ( ) & 1 & 20 & III & 16 & 0) & 02 & 21 & III & 4 & 18 & 2) & 1 & $i$ & 2 & 2 & & 1 & & & 0.3 & \\
\hline$<$ & 6 & (12 & 0) & 2 & 2 & 2 & c) & 1 & 20 & III & 16 & D) & 02 & 21 & III & 4 & 18 & 2) & 1 & & 4 & 0 & 0 & 1 & & & 5 & \\
\hline$<$ & 6 & (12 & $0)$ & 2 & 2 & 0 & c. & 1 & 20 & II I & 12 & 2) & 20 & 1 & III & 4 & 18 & 2) & 1 & 1 & 0 & 0 & & 1 & 0 & 5 & & \\
\hline & 6 & 112 & n) & 2 & 1 & 2 & c 1 & 1 & & III & 12 & 2) & 20 & 1 & III & 4 & i & 2 & 1 & i & 2 & 2 & & 9 & & 0 & $=-0.848$ & \\
\hline$<$ & 6 & (12 & o) & 2 & & & & & & III & $i 2$ & 2) & & 21 & III & 4 & & & 1 & & & & & & & & $=1.03923$ & $(1.08000)$ \\
\hline
\end{tabular}

